\section{Effective leadership and decision- making in animal groups on the move}

\author{
lain D. Couzin ${ }^{1,2}$, Jens Krause ${ }^{3}$, Nigel R. Franks ${ }^{4}$ \& Simon A. Levin
}

${ }^{1}$ Department of Ecology and Evolutionary Biology, Princeton University, Princeton, New Jersey 08544, USA

${ }^{2}$ Department of Zoology, South Parks Road, University of Oxford, Oxford OX1 3PS, UK

${ }^{3}$ Centre for Biodiversity and Conservation, School of Biology, University of Leeds, Leeds LS2 9JT, UK

${ }^{4}$ Centre for Behavioural Biology, School of Biological Sciences, University of

Bristol, Woodland Road, Bristol BS8 1UG, UK

For animals that forage or travel in groups, making movement decisions often depends on social interactions among group members ${ }^{1,2}$. However, in many cases, few individuals have pertinent information, such as knowledge about the location of a food source $^{3,4}$, or of a migration route ${ }^{5-9}$. Using a simple model we show how information can be transferred within groups both without signalling and when group members do not know which individuals, if any, have information. We reveal that the larger the group the smaller the proportion of informed individuals needed to guide the group, and that only a very small proportion of informed individuals is required to achieve great accuracy. We also demonstrate how groups can make consensus decisions, even though informed individuals do not know whether they are in a majority or minority, how the quality of their information compares with that of others, or even whether there are any other informed individuals. Our model provides new insights into the mechanisms of effective leadership and decision-making in biological systems.

Primary questions concerning the mechanism of information transfer in groups include how uninformed individuals recognize those that are informed, whether such recognition is actually necessary, and how groups can come to a collective decision when informed individuals differ in preference ${ }^{10}$. It is known that several animal species have evolved specific recruitment signals that help guide conspecifics. Most famous in this context is the waggle-dance of the honeybee that recruits hive members to visit food sources ${ }^{5,7,8,11}$. Furthermore, valuable experience may be correlated with age or dominance ${ }^{1,2}$, which can presumably be estimated by conspecifics of some species ${ }^{12}$. However, it remains questionable whether such explanations hold when migrating groups of fish, ungulates, insects and birds are considered, where crowding limits the range over which individuals can detect one another ${ }^{1,2}$. In pelagic fish schools, for example, individuals are usually less than one body-length apart ${ }^{13}$. Although it is likely that some species have a genetically determined propensity to migrate in a general direction $^{14,15}$, or respond to abiotic cues such as thermal gradients that may aid migration ${ }^{16,17}$, it is likely for many species that experienced group members play an important role in guiding those that are less experienced or inexperienced. Relatively few informed individuals within fish schools are known to be able to influence the foraging behaviour of the group ${ }^{3}$ and the ability of a school to navigate towards a target ${ }^{4}$. Similarly, very few individuals (approximately 5\%) within honeybee swarms can guide the group to a new nest site?

Furthermore, for some animal groups such as large insect swarms or fish schools, it may be unreasonable to assume that group members have the capacity for individual recognition. Here we address two fundamental issues, both occurring in the absence of complex signalling mechanisms and when it is not possible for group members to establish who has and has not got information. First, how information about the location of resources, or of a migration route, can be transferred within groups; and second, how individuals can achieve a consensus when informed individuals differ in their preferences.

We take into account the ability of grouping individuals to modify their motion on the basis of that of local neighbours (social interactions) $)^{2,18,19}$. Groups are composed of $N$ individuals. Each individual with position vector $\mathbf{c}_{i}(t)$, direction vector $\mathbf{v}_{i}(t)$, and speed $s_{i}$, attempts to maintain a minimum distance $\alpha$ between itself $i$ and others $j$ at all times by turning away from neighbours within that range

$$
\mathbf{d}_{i}(t+\Delta t)=-\sum_{j \neq i} \frac{\mathbf{c}_{j}(t)-\mathbf{c}_{i}(t)}{\left|\left(\mathbf{c}_{j}(t)-\mathbf{c}_{i}(t)\right)\right|}
$$

where $\mathbf{d}_{i}$ represents a desired direction of travel. This simulates individuals acting to maintain personal space and to avoid collisions ${ }^{1,2}$. Avoidance is the highest priority. If neighbours are not detected within this region then the individual will tend to become attracted towards, and aligned with ${ }^{1,2,11,13,18}, j$ neighbours within a local interaction range $\rho$ :

$$
\mathbf{d}_{i}(t+\Delta t)=\sum_{j \neq i} \frac{\mathbf{c}_{j}(t)-\mathbf{c}_{i}(t)}{\left|\left(\mathbf{c}_{j}(t)-\mathbf{c}_{i}(t)\right)\right|}+\sum_{j=1} \frac{\mathbf{v}_{j}(t)}{\left|\mathbf{v}_{j}(t)\right|}
$$

Here $\mathbf{d}_{i}(t+\Delta t)$ is converted to the corresponding unit vector $\hat{\mathbf{d}}_{i}(t+$ $\Delta t)=\mathbf{d}_{i}(t+\Delta t) /\left|\mathbf{d}_{i}(t+\Delta t)\right|$.

To incorporate the influence of informed group members, a proportion of the individuals $p$ are given information about a preferred direction (simulated as a unit vector $\mathbf{g}$ ) representing, for example, the direction to a known resource, or a segment of a migration route. All other individuals are naive and have no preference to move in any particular direction, and are also not informed as to which individuals within the group have information and which do not. Informed individuals balance the influence of their preferred direction and their social interactions with weighting term $\omega$, and replacing $\hat{\mathbf{d}}_{i}(t+\Delta t)$ by $\mathbf{d}_{i}{ }^{\prime}(t+\Delta t)$, where:

$$
\mathbf{d}_{i}{ }^{\prime}(t+\Delta t)=\frac{\hat{\mathbf{d}}_{i}(t+\Delta t)+\omega \mathbf{g}_{i}}{\left|\hat{\mathbf{d}}_{i}(t+\Delta t)+\omega \mathbf{g}_{i}\right|}
$$

If $\omega=0$, vector $\mathbf{g}_{i}$ has no influence and individuals have no desire to move in any specific direction. As $\omega$ approaches 1 , individuals tend to balance their preference to move in direction $\mathbf{g}_{i}$ with their desire to maintain social interactions with group members. As $\omega$ exceeds 1 , individuals are more heavily influenced by their preferred direction $\mathbf{g}_{i}$ than by their neighbours (see Methods section for details of the model).

The accuracy of the group, and hence the quality of information transfer, can be quantified as the normalized angular deviation ${ }^{20}$ of group direction (see Methods) around the preferred direction $\mathbf{g}$, with a minimum value of 0 and maximum of 1 .

For a given group size we found that the accuracy of group motion (in a preferred direction) increased asymptotically as the proportion of informed individuals increased. Furthermore, as group size became larger this relationship became increasingly nonlinear (Fig. 1a), meaning that the larger the group, the smaller the proportion of informed individuals needed to guide the group with a given accuracy. Thus for sufficiently large groups only a very small proportion of informed individuals is needed to achieve close to maximal accuracy. For animal groups such as migrating honeybee colonies, there are likely to be costs associated with increasing the proportion of scouts (informed individuals) within the colony owing to the time taken to recruit others via the waggle-dance ${ }^{5-9,11}$, and for scouts to learn essential navigational skills ${ }^{7,8}$. Thus, we may expect colonies to have evolved such that they have effectively reached the asymptote of accuracy (Fig. 1a) and we predict that they would achieve little benefit in having larger scout populations.

The influence of the weighting of preferred direction $\omega$ was of least importance if the proportion of informed individuals is small 
or large (Fig. 2a, c, d). At intermediate values, however, $\omega$ becomes strongly positively correlated with group accuracy (Fig. 2b). This corresponds to the region in Fig. 1 where the rate of increase in accuracy reaches its asymptote. There is a cost to increasing $\omega$ for these intermediate values of $p$, however, because there exists a tradeoff between the accuracy of group motion and the probability that the group will fragment (Fig. 2b). Lack of group cohesion would clearly be detrimental to honeybees ${ }^{6-8}$ or obligate schooling fish ${ }^{1,2,13}$ and there is experimental evidence that such a trade-off also occurs in other animal groups where social learning of routes depends on the ability of informed individuals to maintain social interactions with those that are naive ${ }^{4}$

Informed individuals within a group may differ in their preferred direction, however, due to differences in experience or motivation $^{1,5-11}$. Groups of animals often have to make collective decisions, such as to move together to a specific resource, such as a nest site ${ }^{1,5-9}$ or food source ${ }^{2,4}$. The means by which such decisions can be made is very poorly understood, especially in the case of large groups and when individuals are not capable of knowing whether they are in a majority or minority, or how the quality of their information compares with that of others, or even whether there are any other individuals in the population with information. To investigate this we created two subsets of informed individual, each having its own directional preference.

If the number of individuals that exhibit each preference is equal, the direction of group motion depends on the degree to which the preferred preferences differ: as this difference increases, groups change from moving in the average preferred direction of all informed individuals to selecting randomly one of the two preferred
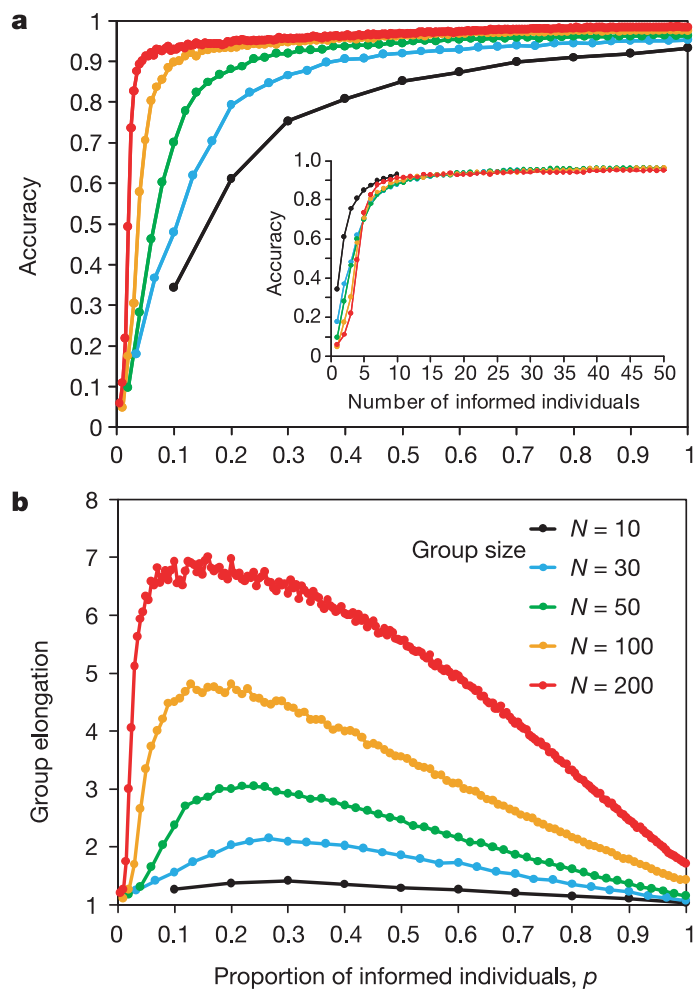

Figure 1 Group accuracy, and shape, as a function of the proportion of informed individuals $p$, for different group sizes $N$. The initial increase in accuracy as a function of $p$ (a) is associated with the group becoming elongated as $p$ increases $(\mathbf{b})$. Informed individuals tend to occupy a frontal position within the group. The elongation of the group then decreases as $p$ increases further and an increasingly large proportion of the group have knowledge of the directional vector $\mathbf{g}$. 400 replicates, $\omega=0.5 ; \alpha=1, \rho=6$, $\gamma=0, \Delta t=0.2 \mathrm{~s}, \theta=2, s_{i}=\alpha \mathrm{s}^{-1}$ corresponding to fish ${ }^{2,19}$. directions (Fig. 3A, a). If the number of individuals exhibiting one of the preferences is increased, however (Fig. $3 \mathrm{~A}, \mathrm{~b}$ and c), the group will (given the appropriate difference in preference, Fig. 3A, a) select collectively the direction preferred by the majority, even if that majority is small (Fig. 3A, b).

This ability of a group to average preferences when differences are relatively small ${ }^{21}$ but to achieve a consensus for the majority option when differences are large is likely to be important in many animal groups, especially if the point at which this transition occurs can be tuned. To achieve this tuning we introduced a simple feedback on the weighting of the preferred direction $\omega$. If, in a given time step, informed individuals find themselves moving in a similar direction (here within a 20-degree arc) to their preferred direction, $\omega$ is reinforced (by $\omega_{\text {inc }}$, up to a maximum, $\omega_{\max }$ ), otherwise it is reduced (by $\omega_{\text {dec }}$, to a minimum of 0 ). Such a feedback loop allows consensus decisions (as opposed to averaging) for smaller differences in preferred direction (Fig. 3B, C). Figure 3B, C shows that the transition to consensus decision-making occurs for small changes in the number of individuals in each subset and that the influence of the minority subset decreases rapidly as the difference in size of the informed subsets increases.

This decision-making mechanism also allows discrimination with respect to quality of information. For example, if there is no difference in the size of the two subsets of informed individual, but there exists a difference in their ability to correctly determine their

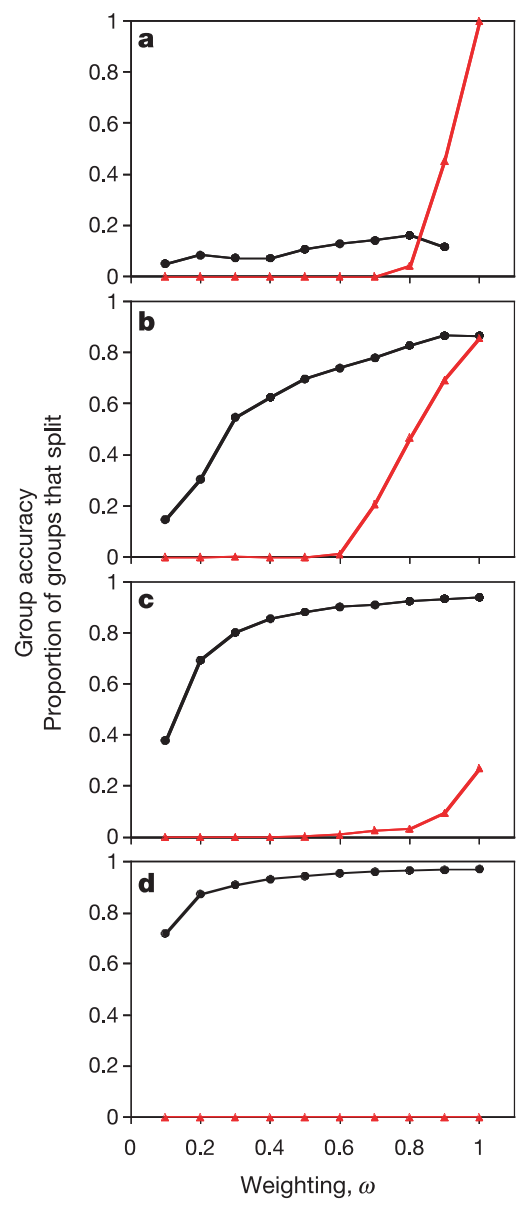

Figure 2 The influence of the weighting of preferred direction. The accuracy of group motion (black circles) and probability of group fragmentation (red triangles) as a function of weighting $\omega$ and the proportion of informed individuals $p$. $\mathbf{a}, p=0.02$ ( 1 individual); b, $p=0.1$ (5 individuals); c, $p=0.2$ (10 individuals); $\mathbf{d}, p=0.5$ (25 individuals). Parameters as for Fig. 1, $N=50$. 


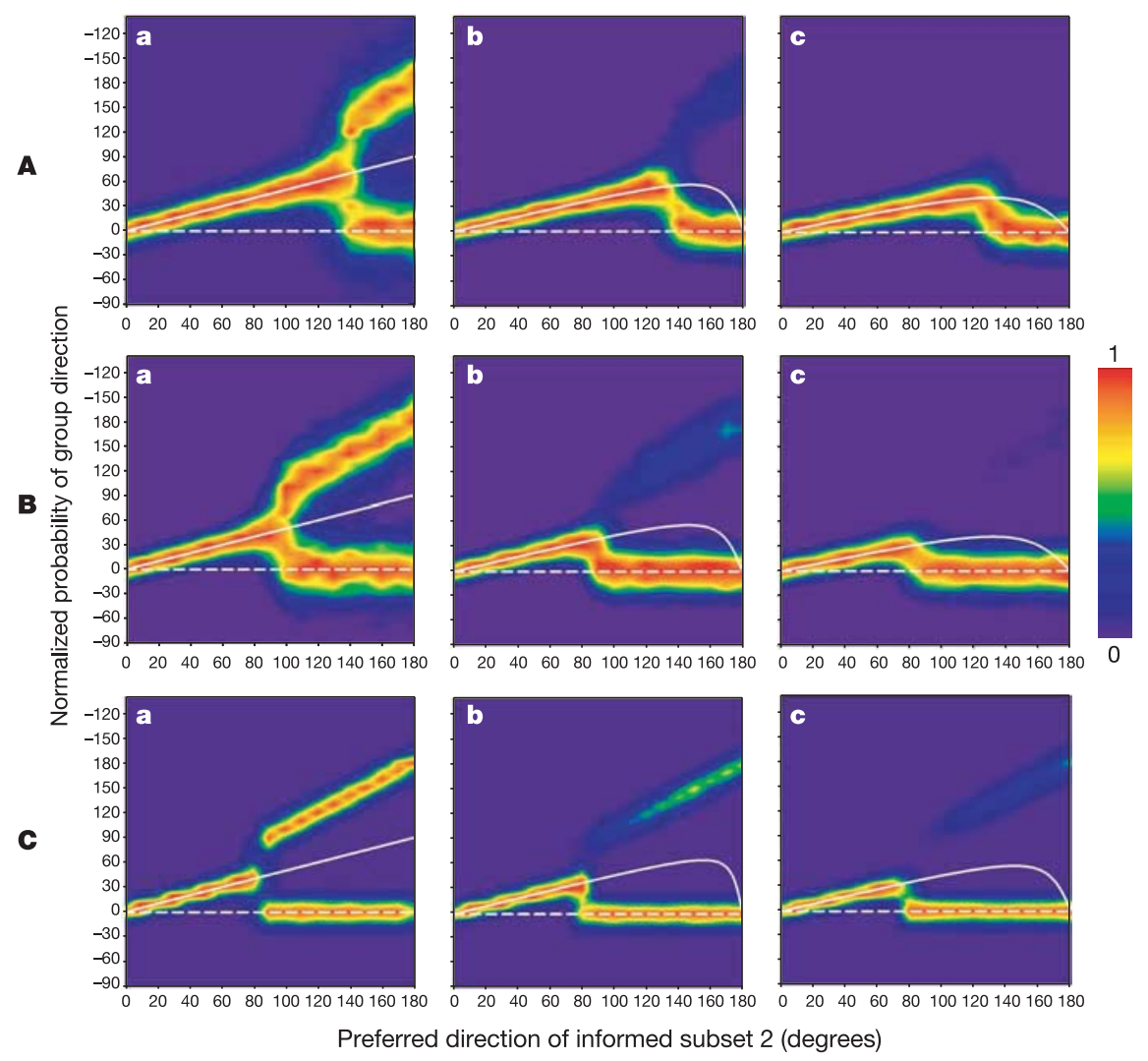

Figure 3 Collective selection of group direction when informed individuals differ in preference. Normalized probability distribution (proportion of maximum) of group direction for groups containing two subsets (with directional preferences $s 1$ and $s 2$, and of sizes $n 1$ and $n 2$, respectively) of informed individual within each group, each with its own directional preference: $s 1=0$ degrees (white dotted line) whereas $s 2=0-180$ degrees (2,000 replicates per 10-degree interval). Total group size $N=100$ (parameters as for Fig. 1; $\omega, \omega_{\max }=0.4$ ). We show the large influence of slightly changing $n 1$ and $n 2$ on group direction. In $\mathbf{a}$, the first column, $n 1=n 2(\mathbf{A}, \mathbf{B}=5 ; \mathbf{C}=10$, demonstrating

preferred direction, the group can select collectively the direction associated with least error (Fig. 4, see Methods for details).

Our model demonstrates that efficient transfer of information, and decision-making, can occur within animal groups in the absence of explicit signals or complex mechanisms for information transfer. This means that informed and naive individuals do not have to be able to recognize each other and that leadership can emerge as a function of information differences among members of

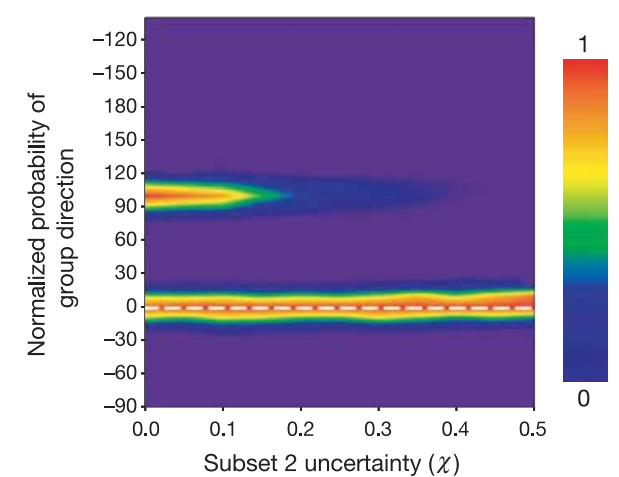

Figure 4 Discrimination between two directions ( $s 1=0 ; s 2=100$ degrees) based on information quality ( $\chi$, see Methods). Parameters as for Fig. 3. $n 1=10, n 2=10$. Subset 2 has uncertain information when $\chi>0$. consistency at larger values of $p$ ). In $\mathbf{b}$, the second column, $n 1$ is increased by 1 , whereas in c, column $3, n 1$ is increased by 1 and $n 2$ decreased by 1 (to allow direct comparison with the first column). B and $\mathbf{C}$ include feedback. $\mathbf{A}, \mathbf{a}, n 1=5, n 2=5 ; \mathbf{A}, \mathbf{b}, n 1=6$, $n 2=5 ; \mathbf{A}, \mathbf{c}, n 1=6, n 2=4 ; \mathbf{B}, \mathbf{a}-\mathbf{c}, n 1$ and $n 2$ as for $\mathbf{A}$, a-c, respectively. $\mathbf{C}, \mathbf{a}, n 1=10, n 2=10 ; \mathbf{C}, \mathbf{b}, n 1=11, n 2=10 . \mathbf{C}, \mathbf{c}, n 1=11, n 2=9$. For $\mathbf{B}$ and $\mathbf{C}$ $\omega_{\text {inc }}=0.012, \omega_{\text {dec }}=0.0008$. Solid white lines are for reference only, representing the direction of the average vector $\mathbf{g}$ of all informed individuals (with constant $\omega$ ).

a population, and is therefore transferable. No inherent differences between individuals (such as dominance due to larger body size) need to be invoked to explain leadership, although these properties can also influence group motion ${ }^{2,18}$. Furthermore, the mechanism of coordination we propose here requires only limited cognitive ability, and demonstrates that individuals can respond spontaneously to those that have information. This is important to our understanding of group foraging, social learning, migration and navigation, and may provide new design protocols for information transfer among grouping robots.

\section{Methods}

Motion in animals is subject to random influences (for example, sensory/movement error). This is simulated by modifying $\hat{\mathbf{d}}_{i}(t+\Delta t)$ or $\mathbf{d}_{i}{ }^{\prime}(t+\Delta t)$, for uninformed and informed individuals, respectively, by rotating it by a random angle taken from a circularwrapped gaussian distribution, centred on 0 , with standard deviation $\sigma=0.01$ radians (see 'Uncertainty of information', below) resulting in vector $\mathbf{d}_{i}{ }^{\prime \prime}(t+\Delta t)$. Individuals can turn through an angle of, at most, $\theta \Delta t$ radians towards their desired direction in time $\Delta t$; if the angle between $\mathbf{v}_{i}(t)$ and $\mathbf{d}_{i}{ }^{\prime \prime}(t+\Delta t)$ is less than $\theta \Delta t$, they achieve alignment with their desired vector, $\mathbf{v}_{i}(t+\Delta t)=\mathbf{d}_{i}{ }^{\prime \prime}(t+\Delta t)$, otherwise they turn $\theta \Delta t$ towards it. The new position vector of individual $i$ is then given by $\mathbf{c}_{i}(t+\Delta t)=\mathbf{c}_{i}(t)+\mathbf{v}_{i}(t+\Delta t) \Delta t s_{i}$, where $s_{i}$ is the speed of individual $i$.

\section{Group size}

Group sizes here are comparable to the size of schools, flocks or herds, of many species $^{1-4,13,19}$, but smaller than large aggregates such as honeybee colonies ${ }^{7,8}$, owing to the nonlinear increase in computer processing time required as $N$ increases. Our results, however, are likely to be independent of absolute group size, within the constraints of maintaining cohesion of group members ${ }^{22}$. To automatically test whether groups remained cohesive we used the equivalence class technique described in refs 18, 19. 


\section{Group direction}

To quantify group direction $\mathbf{h}$ we create a vector extending from the group's centroid calculated at time $t_{f} \Delta t-50 \Delta t$ to the centroid calculated at $t_{f} \Delta t$, where $t_{f}$, the final time step, is 2,500. In Figs 1 and 2 we calculated the mean angular deviation $s$ for 400 replicates, equivalent to calculating the linear standard deviation ${ }^{20}$, which we normalized so that its minimum value is 0 , corresponding to no information transfer (groups move in random directions), and its maximum value is 1 , corresponding to the motion of the simulated groups always being exactly aligned with $\mathbf{g}$.

\section{Elongation}

Elongation was measured by creating a bounding box around the group aligned with the direction of travel and calculating the ratio of the length of the axis aligned with the group direction, to that perpendicular to group direction. This value is 1 when both axes are identical, $>1$ as the group becomes more elongated in the direction of travel, and $<1$ as it becomes elongated perpendicular to the direction of travel.

\section{Uncertainty of information}

Individuals may also not have perfect knowledge about their preferred direction $\mathbf{g}$, and this can be simulated for each individual $i$ at the start of the simulation by rotating by the same type of circular-wrapped gaussian distribution with standard deviation $\gamma$, resulting in vector $\mathrm{g}_{i}$. Changing $\gamma$ changes our results quantitatively, but not qualitatively, within the upper limits imposed by groups being able to maintain cohesion (see Supplementary Fig. 1).

To simulate a difference in the ability of informed individuals, within the same group, to correctly determine their preferred direction, $\mathbf{g}$ is rotated for $p / 2$ individuals $(s 2)$ by gaussian-distributed angle, with standard deviation $\chi$ radians (creating $\mathbf{g}_{i}$, as above) at the start of the simulation (see Fig. 4).

\section{Starting conditions}

Each simulation run was started with randomized individual positions and orientations.

1. Krause, J. \& Ruxton, G. D. Living in Groups 84-85, 137-143 (Oxford Univ. Press, Oxford, 2002).

2. Couzin, I. D. \& Krause, J. Self-organization and collective behaviour in vertebrates. Adv. Study Behav. 32, 1-75 (2003).

3. Reebs, S. G. Can a minority of informed leaders determine the foraging movements of a fish shoal? Anim. Behav. 59, 403-409 (2000).

4. Swaney, W., Kendal, J., Capon, H., Brown, C. \& Laland, K. N. Familiarity facilitates social learning of foraging behaviour in the guppy. Anim. Behav. 62, 591-598 (2001).

5. Franks, N. R., Pratt, S. C., Mallon, E. B., Britton, N. F. \& Sumpter, D. J. T. Information flow, opinion polling and collective intelligence in house-hunting social insects. Phil. Trans. R. Soc. Lond. B 357, 1567-1583 (2002)

6. Lindauer, M. Communication in swarm-bees searching for a new home. Nature 179, $63-66$ (1957).

7. Seeley, T. D. Honeybee Ecology: a Study of Adaptation in Social Life 71-74 (Princeton Univ. Press, Princeton, 1985)

8. Seeley, T. D. The Wisdom of the Hive 34-35 (Harvard Univ. Press, Cambridge, 1995).

9. Seeley, T. D. Consensus building during nest-site selection in honey bee swarms: the expiration of dissent. Behav. Ecol. Sociobiol. 53, 417-424 (2003).

10. Conradt, L. \& Roper, T. J. Group decision-making in animals. Nature 421, 155-158 (2003).

11. von Frisch, K. The Dance Language and Orientation of Bees 28-235 (Harvard Univ. Press, Harvard, 1967).

12. Rubenstein, D. I. \& Hack, M. Horse signals: the sounds of scents and fury. Evol. Ecol. 6, 254-260 (1992).

13. Partridge, B. L. Structure and function of fish schools. Sci. Am. 245, 114-123 (1982).

14. Berthold, P. \& Querner, U. Genetic basis of migratory behaviour in European warblers. Science 212, $77-79$ (1981).

15. Berthold, P., Helbig, A. J., Mohr, G. \& Querner, U. Rapid microevolution of migratory behaviour in a wild bird species. Nature 360, 668-670 (1992).

16. Neill, W. H. Mechanisms of fish distribution in heterothermal environments. Am. Zool. 19, 305-317 (1979).

17. Grunbaum, D. Schooling as a strategy for taxis in a noisy environment. Evol. Ecol. 12, 503-522 (1998).

18. Couzin, I. D., Krause, J., James, R., Ruxton, G. D. \& Franks, N. R. Collective memory and spatial sorting in animal groups. J. Theor. Biol. 218, 1-11 (2002).

19. Hoare, D. J., Couzin, I. D., Godin, J.-G. \& Krause, J. Context-dependent group-size choice in fish. Anim. Behav. 67, 155-164 (2004).

20. Batschelet, E. Circular Statistics in Biology 34-36 (Academic, London, 1981).

21. Simons, A. M. Many wrongs: the advantage of group navigation. Trends Ecol. Evol. 19, 453-455 (2004).

22. Gregóire, G., Chaté, H. \& Tu, Y. Moving and staying together without a leader. Physica D 181, 157-170 (2003).

Acknowledgements I.D.C. thanks the Pew Charitable Trusts, the NSF and the EPSRC for their support. I.D.C. and J.K. acknowledge an EPSRC grant and are also grateful for fellowships at the Centre for Interdisciplinary Research, University of Bielefeld, where we had the opportunity to develop this research. S.A.L. acknowledges support from the NSF and the Andrew W. Mellon Foundation, and N.R.F. from the EPSRC and the BBSRC. I.D.C. thanks Balliol College for support and S. Pratt, D. Rubenstein, D. James and A. Ward for their input.

Competing interests statement The authors declare that they have no competing financial interests.

Correspondence and requests for materials should be addressed to I.D.C.

(iain.couzin@zoo.ox.ac.uk or icouzin@princeton.edu). 